See discussions, stats, and author profiles for this publication at: https://www.researchgate.net/publication/325181154

\title{
Track-Down Operations on Bilattices
}

Conference Paper · May 2018

DOI: 10.1109/ISMVL.2018.00021

CITATIONS

READS

0

1 author:

Damian Szmuc

(2)

IIF-SADAF, CONICET and University of Buenos Aires

10 PUBLICATIONS 18 CITATIONS

SEE PROFILE

Some of the authors of this publication are also working on these related projects:

Weak self-referential solutions to paradoxes View project

Generalizations of the Weak Kleene logical framework View project 


\title{
Track-Down Operations on Bilattices
}

\author{
Damian Szmuc ${ }^{1,2}$ \\ ${ }^{1}$ IIF-SADAF, National Scientific and Technical Research Council (CONICET), Argentina \\ 2 Department of Philosophy, University of Buenos Aires, Argentina
}

\begin{abstract}
This paper discusses a dualization of Fitting's notion of a "cut-down" operation on a bilattice, rendering a "track-down" operation, later used to represent the idea that a consistent opinion cannot arise from a set including an inconsistent opinion. The logic of track-down operations on bilattices is proved equivalent to the logic $\mathrm{dS}_{\text {fde }}$, dual to Deutsch's system $\mathrm{S}_{\text {fde }}$. Furthermore, track-down operations are employed to provide an epistemic interpretation for paraconsistent weak Kleene logic. Finally, two logics of sequential combinations of cut- and track-down operations allow settling positively the question of whether bilattice-based semantics are available for subsystems of $S_{f d e}$.
\end{abstract}

\section{Introduction: Aim and Definitions}

This paper discusses a dualization of Fitting's notion of a "cut-down" operation on a bilattice, used in [9] to provide an epistemic interpretation of Kleene's paracomplete weak three-valued logic. The logic of such cut-down operations is equivalent, as shown in [7], to Deutsch's fourvalued logic $S_{\text {fde }}$ from [5]. Our dualization of Fitting's notion renders a "track-down" operation later used to represent the idea that a consistent opinion cannot arise from a set including an inconsistent opinion.

Our first result in this paper is proving the logic of track-down operations is equivalent to the four-valued logic $\mathrm{dS}_{\text {fde }}$, dual to Deutsch's system. Our second contribution consists in employing such track-down operations to provide an epistemic interpretation for paraconsistent weak Kleene logic. Our third contribution is settling positively the question, posed in [7], whether bilattice-based semantics can be given for subsystems of $S_{\text {fde }}$. This is done by presenting two subsystems of $\mathrm{S}_{\text {fde }}$ and $\mathrm{dS}_{\text {fde }}$ corresponding to the logics of two different sequential combinations of cut- and track-down operations.

In what follows, $\mathcal{L}$ will be the propositional language $\{\dot{\rightarrow}, \dot{\wedge}, \dot{V}\}$ and $F O R(\mathcal{L})$ the set of formulae of $\mathcal{L}$, defined as usual. Formulae of $\mathcal{L}$ will be denoted by $\varphi, \psi$, etc., while sets of formulae will be denoted by $\Gamma, \Delta$, etc. For a propositional language $\mathcal{L}$, a matrix $\mathcal{M}$ is a structure $\langle\mathcal{V}, \mathcal{D}, \mathcal{O}\rangle$ where $\langle\mathcal{V}, \mathcal{O}\rangle$ is an algebra of the same similarity type as $\mathcal{L}$, and $\mathcal{D}$ is a non-empty proper subset of $\mathcal{V}$. Given $\mathcal{M}$, a valuation $v$ is a homomorphism from $F O R(\mathcal{L})$ to $\mathcal{V}$. A (matrix) logic $\mathrm{L}$ is a pair $\left\langle F O R(\mathcal{L}), \vDash_{\mathcal{M}}\right\rangle$ where $\vDash_{\mathcal{M}} \subseteq \wp(F O R(\mathcal{L})) \times F O R(\mathcal{L})$ is a substitution-invariant consequence relation defined by letting $\Gamma \vDash_{\mathcal{M}} \varphi$ iff for every valuation $v$, if $v[\Gamma] \subseteq \mathcal{D}$, then $v(\varphi) \in \mathcal{D}$. When $\mathrm{L}=\left\langle F O R(\mathcal{L}), \vDash_{\mathcal{M}}\right\rangle$ we may denote $\vDash_{\mathcal{M}}$ by $\vDash_{\mathrm{L}}$. 


\section{Bilattices, Cut-downs and Track-downs}

Definition 1 (Ginsberg [10]). A pre-bilattice $\mathcal{B}$ is a structure $\left\langle B, \leq_{k}, \leq_{t}\right\rangle$ such that $B$ is a nonempty set and $\left\langle B, \leq_{k}\right\rangle,\left\langle B, \leq_{t}\right\rangle$ are two complete lattices. ${ }^{1}$

Definition 2 (Ginsberg [10]). A bilattice $\mathcal{B}$ is a structure of the form $\left\langle B, \leq_{k}, \leq_{t}, \neg\right\rangle$ such that $\left\langle B, \leq_{k}, \leq_{t}\right\rangle$ is a pre-bilattice and $\neg$ is an involutive $t$-inverting function on $B$, i.e. $\neg: B \longrightarrow B$ is a function such that for all $a, b \in B$ : (i) $\neg \neg a=a$, (ii) If $a \leq_{k} b$, then $\neg a \leq_{k} \neg b$, (iii) If $a \leq_{t} b$, then $\neg b \leq_{t} \neg a$.

The orders $\leq_{k}$ and $\leq_{t}$ are often referred to as the "information" (sometimes, "knowledge") ordering, and the "truth" ordering, respectively. The lattice-theoretic operations meet and join related to these orderings are, respectively, $\otimes$ and $\oplus$, and the usual $\wedge$ and $\vee$.

Fitting famously offered numerous epistemic motivations to work with bilattices. For instance, in [9] he proposes to consider a group $\mathcal{E}$ of experts whose opinion we value and who we are consulting on certain matters, in the form of a series of yes/no questions. When asking these experts about a certain sentence $\varphi$ some will say it is true, some will say it is false, some may be willing to decline expressing an opinion and some may have reasons for calling it both true and false.

To such a scenario corresponds, Fitting claims, the assignment of a sort of generalized truthvalue to $\varphi$, namely $v(\varphi)=\langle P, N\rangle$ where $P$ is the set of experts who claim that $\varphi$ is true, and $N$ is the set of experts who claim that $\varphi$ is false. Given this, it is possible that $P \cup N \neq \mathcal{E}$ and it is also possible that $P \cap N \neq \emptyset$. As Fitting notices

Orderings can be introduced into our people-based structure: set $\left\langle P_{1}, N_{1}\right\rangle \leq_{k}\left\langle P_{2}, N_{2}\right\rangle$ if $P_{1} \subseteq P_{2}$ and $N_{1} \subseteq N_{2}$, and set $\left\langle P_{1}, N_{1}\right\rangle \leq_{t}\left\langle P_{2}, N_{2}\right\rangle$ if $P_{1} \subseteq P_{2}$ and $N_{2} \subseteq N_{1}(\ldots)$ Thus, information goes up if more people express a positive or negative opinion, and truth goes up if people drop negative opinions or add positive ones. This gives a structure of a pre-bilattice. $[9$, p. 3$]$

In such a framework, let us additionally refer to the generalized truth-values $\langle\mathcal{E}, \emptyset\rangle,\langle\mathcal{E}, \mathcal{E}\rangle$, $\langle\emptyset, \emptyset\rangle$ and $\langle\emptyset, \mathcal{E}\rangle$ with the labels $\mathbf{t}, \top, \perp, \mathbf{f}$, respectively. Focusing in the $\{\mathbf{t}, \top, \perp, \mathbf{f}\}$-reduct of this structure renders the famous bilattice $\mathcal{F O U R}$.

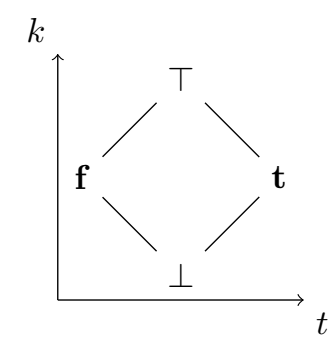

Figure 1: The bilattice $\mathcal{F O U R}$

By considering situations where the generalized truth-values assigned to any sentence are elements of $\mathcal{F O U R}$ (or some subset thereof) Fitting was able to provide an epistemic interpretation of several Kleene logics. In fact, in [9], the way the connectives $\dot{\rightarrow}, \dot{\wedge}, \dot{\vee}$ work in the strong

\footnotetext{
${ }^{1}$ There is no need for completeness to be built into the definition of a bilattice. We assume it here, though, to keep consistency with the literature.
} 
Kleene logic $\mathrm{K}_{3}$ and its four-valued generalization $\mathrm{E}_{\text {fde }}$ due to Belnap and Dunn in [3] and [6] are interpreted in terms of the bilattice operations $\neg, \wedge, \vee$ on $\mathcal{F O U R}$.

Our target in this paper are, nevertheless, not the strong but the weak Klenee logics and some subsystems thereof. Employing Fitting's framework to provide an epistemic interpretation of the operations of Kleene's weak three-valued logic requires some subtleties. More precisely, Fitting said in [9, pp. 66-67] that sometimes, e.g. when evaluating a conjunction $\varphi \dot{\wedge} \psi$ or a disjunction $\varphi \dot{\vee} \psi$, we may want to

'cut this down' by considering people who have actually expressed an opinion on both propositions $[\varphi]$ and $[\psi]$

Whence, we shall call the resulting alternative conjunctions and disjunctions-following Ferguson in [7] - the "cut-down variants" of these famous logical operations. Observe $\neg$ is not altered by this cut-down policy, as remarked in $[9$, p. 67] and [7, p. 24].

Definition 3 (Fitting [9]). For an element $a \in B$, the Kleene-Fitting cut-down of $a$, denoted by $\llbracket a \rrbracket$ is defined as $a \oplus \neg a$

To this extent, the cut-down $\llbracket \varphi \rrbracket$ of a $\varphi$ is intended to output "those who either think $\varphi$ is true, or think $\varphi$ is false".

Definition 4 (Fitting [9]). For $a, b \in B$, the Kleene-Fitting cut-down operations $\Delta$ and $\nabla$ are defined as:

$$
a \triangle b=(a \wedge b) \otimes \llbracket a \rrbracket \otimes \llbracket b \rrbracket \quad a \nabla b=(a \vee b) \otimes \llbracket a \rrbracket \otimes \llbracket b \rrbracket
$$

Similarly, the cut-down variants of a conjunction and disjunction shall be interpreted as cutting down the set of experts under consideration, to only those who have expressed an opinion towards all of the propositions involved. These variants works so that no determinate opinion on e.g. $\varphi \dot{\wedge} \psi$ or $\varphi \dot{\vee} \psi$ can arise from a set that includes an indeterminate opinion on $\varphi$ or $\psi$.

In [9], the way the connectives $\dot{\Lambda}, \dot{V}$ work in the weak Kleene logic $\mathrm{K}_{3}^{w}$ was interpreted in terms of the cut-down operations $\Delta, \nabla$, providing the target epistemic interpretation.

Definition 5. $\mathrm{K}_{3}^{w}$ is the three-valued logic induced by the matrix $\left\langle\mathcal{V}_{\mathrm{K}_{3}^{w}}, \mathcal{D}_{\mathrm{K}_{3}^{w}}, \mathcal{O}_{\mathrm{K}_{3}^{w}}\right\rangle$, where $\mathcal{V}_{\mathrm{K}_{3}^{w}}=\{\mathbf{t}, \perp, \mathbf{f}\}, \mathcal{D}_{\mathrm{K}_{3}^{w}}=\{\mathbf{t}\}, \mathcal{O}_{\mathrm{K}_{3}^{w}}=\left\{\dot{\mathrm{K}_{3}^{w}}, \dot{\hat{K}_{3}^{w}}, \dot{\mathrm{K}}_{3}^{w}\right\}$ and these truth-functions are defined by the truth-tables in Figure 2.

\begin{tabular}{|c|c|c|c|c|c|c|c|c|c|}
\hline & $\mathrm{K}_{3}^{w}$ & $\hat{\mathrm{K}}_{3}^{w}$ & $\mathbf{t}$ & $\perp$ & f & $\begin{array}{l}\dot{V} \\
\mathrm{~K}_{3}^{w}\end{array}$ & $\mathbf{t}$ & $\perp$ & $\mathbf{f}$ \\
\hline $\mathbf{t}$ & $\mathbf{f}$ & $\mathrm{t}$ & $\mathbf{t}$ & $\perp$ & $\mathrm{f}$ & $\mathrm{t}$ & $\overline{\mathbf{t}}$ & $\perp$ & $\mathrm{t}$ \\
\hline$\perp$ & $\perp$ & $\perp$ & $\perp$ & $\perp$ & $\perp$ & $\perp$ & $\perp$ & $\perp$ & $\perp$ \\
\hline f & $\mathbf{t}$ & f & f & $\perp$ & f & f & $\mathbf{t}$ & $\perp$ & $\mathbf{f}$ \\
\hline
\end{tabular}

Figure 2: Truth-tables for $\mathrm{K}_{3}^{w}$

When pooling the opinion of experts, we may want to follow Fitting's cut-down strategy or we may want to proceed in a different, although perfectly dual, way. Sometimes, e.g. when considering a conjunction $\varphi \dot{\wedge} \psi$ or a disjunction $\varphi \dot{v} \psi$ we may want to

'track down' people who have expressed an inconsistent opinion towards either propositions, $\varphi$ or $\psi$ 
Whence, we shall call the resulting alternative conjunctions and disjunctions, the "track-down" variants of these famous logical operations. Observe, again, that $\neg$ is not altered by this cutdown policy, either.

Definition 6. For an element $a \in B$, the track-down of $a$, denoted by $\rrbracket a \llbracket$ is defined as $a \otimes \neg a$

To this extent, the track-down $\rrbracket \varphi \llbracket$ of $\varphi$ is intended to output "those who think $\varphi$ is true, and also think $\varphi$ is false".

Definition 7. For $a, b \in B$, the track-down operations $\boldsymbol{\Delta}$ and $\boldsymbol{\nabla}$ are defined as:

$$
a \mathbf{\Lambda}=(a \wedge b) \oplus \rrbracket a \llbracket \oplus \rrbracket b \llbracket \quad a \mathbf{\nabla}=(a \vee b) \oplus \rrbracket a \llbracket \oplus \rrbracket b \llbracket
$$

Similarly, the track-down variants of a conjunction and disjunction shall be interpreted as tracking down the set of experts that expressed an inconsistent opinion towards some of the propositions involved, and then expanding the set of experts thinking the conjunctions or disjunctions are true, or that they are false, to account for them. These variants works so that no consistent opinion on e.g. $\varphi \dot{\wedge} \psi$ or $\varphi \dot{\vee} \psi$ can arise from a set that includes an inconsistent opinion on $\varphi$ or $\psi$.

In Section 5 we will establish that it is possible to interpret the way the connectives $\dot{\wedge}, \dot{V}$ work in the paraconsistent weak Kleene logic PWK in terms of the track-down operations $\mathbf{\Lambda}, \mathbf{\nabla}$, thereby providing an epistemic interpretation of PWK.

Definition 8. PWK is the three-valued logic induced by the matrix $\left\langle\mathcal{V}_{\mathrm{PWK}}, \mathcal{D}_{\mathrm{PWK}}, \mathcal{O}_{\mathrm{PWK}}\right\rangle$, where $\mathcal{V}_{\mathrm{PWK}}=\{\mathbf{t}, \top, \mathbf{f}\}, \mathcal{D}_{\mathrm{PWK}}=\{\mathbf{t}, \top\}, \mathcal{O}_{\mathrm{PWK}}=\left\{\dot{\overrightarrow{\mathrm{PWK}}}, \dot{\mathrm{PWK}_{\mathrm{PW}},}, \dot{\mathrm{PWK}}\right\}$ and these truth-functions are defined by the truth-tables in Figure 3 .

\begin{tabular}{|c|c|c|c|c|c|c|c|c|c|}
\hline & $\dot{\vec{P}}$ PKK & $\dot{\hat{P}}_{\mathrm{PWK}}$ & $\mathbf{t}$ & $T$ & $\mathbf{f}$ & $\begin{array}{l}\dot{V} \\
\text { PWK }\end{array}$ & $\mathbf{t}$ & $T$ & $\mathbf{f}$ \\
\hline $\mathrm{t}$ & $f$ & $\mathbf{t}$ & $\mathbf{t}$ & $T$ & $\mathbf{f}$ & $\mathrm{t}$ & $\mathbf{t}$ & $T$ & $\mathbf{t}$ \\
\hline$T$ & $T$ & $\top$ & $\top$ & $\top$ & $\top$ & $\top$ & $\top$ & $\top$ & $T$ \\
\hline f & $\mathrm{t}$ & f & f & $\top$ & $\mathbf{f}$ & f & $\mathbf{t}$ & $\top$ & $\mathbf{f}$ \\
\hline
\end{tabular}

Figure 3: Truth-tables for PWK

\section{Four Containment Logics}

The various logics of cut- and track-down operations on bilattices we are going to study in the sequel belong to a peculiar class of logics dubbed containment logics.

These are systems where valid inferences comply with certain set-theoretic containment principles relating the set of propositional variables appearing in the premises and the set of propositional variables appearing in the conclusion.

Among containment logics, the best known systems belong to the family of "Parry" logicsso-called because their valid inferences comply with a form of Parry's Proscriptive Principle for entailment, discussed in [12], namely

$$
\Gamma \vDash_{\mathrm{L}} \varphi \text { only if } \operatorname{var}(\varphi) \subseteq \operatorname{var}(\Gamma)
$$

where $\operatorname{var}(\varphi)$ is the set of propositional variables appearing in $\varphi$. As highlighted in [8], the following is a Parry logic. 


\begin{tabular}{|c|c|c|c|c|c|c|c|c|c|c|c|}
\hline & $\dot{\vec{S}}_{\mathrm{fde}}$ & $\dot{\hat{\Lambda}}$ & $\mathbf{t}$ & $\top$ & $\perp$ & f & $\begin{array}{l}\dot{V} \\
S_{\text {fde }}\end{array}$ & $\mathbf{t}$ & $\top$ & $\perp$ & $\mathbf{f}$ \\
\hline $\mathbf{t}$ & $\mathbf{f}$ & $\mathbf{t}$ & $\mathrm{t}$ & $T$ & $\perp$ & f & $\mathbf{t}$ & $\mathrm{t}$ & $\mathbf{t}$ & $\perp$ & $\mathbf{t}$ \\
\hline$\top$ & $\top$ & $\top$ & $\top$ & $\top$ & $\perp$ & f & $\top$ & $\mathrm{t}$ & $\top$ & $\perp$ & $\top$ \\
\hline$\perp$ & $\perp$ & $\perp$ & $\perp$ & $\perp$ & $\perp$ & $\perp$ & $\perp$ & $\perp$ & $\perp$ & $\perp$ & $\perp$ \\
\hline f & $\mathbf{t}$ & f & f & f & $\perp$ & f & $\mathbf{f}$ & $\mathbf{t}$ & $\top$ & $\perp$ & $\mathbf{f}$ \\
\hline
\end{tabular}

Figure 4: Truth-tables for the logic $S_{\text {fde }}$

Definition 9 (Deutsch [5]). $S_{\text {fde }}$ is the four-valued logic induced by the matrix $\left\langle\mathcal{V}_{\mathrm{S}_{\text {fde }}}, \mathcal{D}_{\mathrm{S}_{\text {fde }}}, \mathcal{O}_{\mathrm{S}_{\text {fde }}}\right\rangle$, where $\mathcal{V}_{\mathrm{S}_{\mathrm{fde}}}=\{\mathbf{t}, \top, \perp, \mathbf{f}\}, \mathcal{D}_{\mathrm{S}_{\mathrm{fde}}}=\{\mathbf{t}, \top\}, \mathcal{O}_{\mathrm{S}_{\mathrm{fde}}}=\left\{\dot{\overrightarrow{\mathrm{f}_{\mathrm{fde}}}},{ }_{\mathrm{S}_{\mathrm{fde}}}, \dot{\mathrm{V}}_{\mathrm{fde}}\right\}$ and these truth-functions are defined by the truth-tables in Figure 4 .

The logic $S_{\text {fde }}^{w}$, semantics for which were first given in [13], is also a Parry logic. This can be established by noting, as we do below, that $S_{\text {fde }}^{w}$ is a fragment of $S_{\text {fde }}$.

Definition 10. $S_{\text {fde }}^{w}$ is the four-valued logic induced by the matrix $\left\langle\mathcal{V}_{S_{\text {fde }}^{w}}^{w}, \mathcal{D}_{\mathrm{S}_{\text {fde }}}^{w}, \mathcal{O}_{\mathrm{S}_{\text {fde }}}^{w}\right\rangle$, where $\mathcal{V}_{\mathrm{S}_{\mathrm{fde}}^{w}}^{w}=\{\mathbf{t}, \top, \perp, \mathbf{f}\}, \mathcal{D}_{\mathrm{S}_{\mathrm{fde}}^{w}}^{w}=\{\mathbf{t}, \top\}, \mathcal{O}_{\mathrm{S}_{\mathrm{fde}}^{w}}=\left\{\dot{\overrightarrow{S_{\mathrm{fde}}^{w}}}, \dot{\mathrm{S}_{\mathrm{fde}}^{w}}, \underset{\mathrm{S}_{\mathrm{fde}}^{w}}{\dot{v}}\right\}$ and these truth-functions are defined by the truth-tables in Figure 5 .

\begin{tabular}{|c|c|c|c|c|c|c|c|c|c|c|c|}
\hline & $\dot{\vec{S}}_{\mathrm{fde}}^{w}$ & $\hat{\Lambda}_{\mathrm{S}_{\text {fde }}^{w}}^{w}$ & $\mathbf{t}$ & $\top$ & $\perp$ & f & $\begin{array}{l}\dot{V}_{S_{\text {fde }}^{w}} \\
\text {. }\end{array}$ & $\mathbf{t}$ & $\top$ & $\perp$ & $\mathbf{f}$ \\
\hline $\mathbf{t}$ & f & $\mathbf{t}$ & $\mathrm{t}$ & $\bar{T}$ & $\perp$ & $f$ & $\mathbf{t}$ & $\mathrm{t}$ & $\mathrm{T}$ & $\perp$ & $\mathbf{t}$ \\
\hline$\top$ & $\top$ & $\top$ & $\top$ & $\top$ & $\perp$ & $\top$ & $\top$ & $\top$ & $\top$ & $\perp$ & $\top$ \\
\hline$\perp$ & $\perp$ & $\perp$ & $\perp$ & $\perp$ & $\perp$ & $\perp$ & $\perp$ & $\perp$ & $\perp$ & $\perp$ & $\perp$ \\
\hline f & $\mathbf{t}$ & $\mathbf{f}$ & $\mathbf{f}$ & $\top$ & $\perp$ & f & $\mathbf{f}$ & $\mathbf{t}$ & $\top$ & $\perp$ & $\mathbf{f}$ \\
\hline
\end{tabular}

Figure 5: Truth-tables for the logic $S_{\text {fde }}^{w}$

That $S_{\text {fde }}^{w}$ is a fragment of $S_{\text {fde }}$ can be observed by showing the definability of the $S_{\text {fde }}^{w}$ truthfunctions in terms of $S_{\text {fde }}$ truth-functions. ${ }^{2}$ Letting the trivial case of negation aside, it can be easily checked, by looking at the corresponding truth-tables, that $\underset{\mathrm{S}_{\mathrm{fde}}^{w}}{\dot{x}}(x, y)$ is definable in $\mathrm{S}_{\mathrm{fde}}$ as

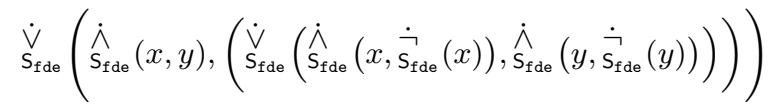

later defining $\underset{S_{\text {fde }}^{w}}{\dot{v}}$ in terms of $\underset{S_{\text {fde }}^{w}}{\dot{x}}$ and $\dot{\overrightarrow{S_{\text {fde }}^{w}}}$, as is customary.

Now, besides Parry logics, there is yet another important and not so widely discussed family of containment logics, which we may naturally refer to as "Dual Parry" logics - for all of their valid inferences comply with a dual of Parry's Proscriptive Principle for entailment, namely

$$
\Gamma \vDash_{\mathrm{L}} \varphi \text { only if } \exists \Gamma^{\prime} \subseteq \Gamma, \Gamma^{\prime} \neq \emptyset, \operatorname{var}\left(\Gamma^{\prime}\right) \subseteq \operatorname{var}(\varphi)
$$

It can be proved (as we do in Observation 1) that the logic defined below, semantics for which were given for the first time in [13], is indeed a Dual Parry logic. ${ }^{3}$

\footnotetext{
${ }^{2}$ This follows the strategy of $[11, \S 5.1]$ to show that Kleene's weak three-valued logics (e.g. $\mathrm{K}_{3}^{w}$ ) are fragments of their strong counterparts (e.g. $\mathrm{K}_{3}$ ) - whence, the addition of the superscript $w$ to these systems.

${ }^{3}$ Notice that the clause $\Gamma^{\prime} \neq \emptyset$ would render enjoying the dual Parry Principle incompatible with having theorems. Thus, a reviewer suggests replacing it with the condition that $\Gamma^{\prime} \vDash_{\mathrm{L}} \varphi$. Although this is an interesting alternative, the incompatibility induced by the current clause is dual - as expected - to the fact that enjoying the Parry Principle is incompatible with having anti-theorems. that is, with there being formulae $\psi$ such that $\psi \vDash_{\mathrm{L}} \emptyset$.
} 


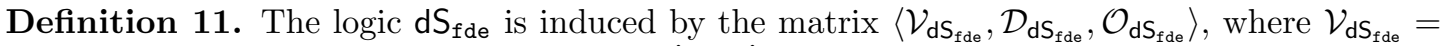
$\{\mathbf{t}, \top, \perp, \mathbf{f}\}, \mathcal{D}_{\mathrm{dS}_{\mathrm{fde}}}=\{\mathbf{t}, \top\}, \mathcal{O}_{\mathrm{dS}_{\mathrm{fde}}}=\left\{\dot{\overrightarrow{d S}}_{\mathrm{fde}}, \dot{\hat{d S}}_{\mathrm{fde}}, \dot{V}_{\mathrm{dS}_{\mathrm{fde}}}\right\}$ and these truth-functions are defined by the truth-tables in Figure 6.

\begin{tabular}{|c|c|c|c|c|c|c|c|c|c|c|c|}
\hline & $\dot{\vec{d}}_{\mathrm{d}}$ & $\dot{\mathrm{dS}}_{\mathrm{dfde}}$ & $\mathbf{t}$ & $\top$ & $\perp$ & $\mathrm{f}$ & $\begin{array}{l}\dot{V} \\
\mathrm{dS}_{\mathrm{fde}}\end{array}$ & $\mathrm{t}$ & T & $\perp$ & $\mathrm{f}$ \\
\hline $\mathrm{t}$ & f & $\mathrm{t}$ & $\mathbf{t}$ & $T$ & $\perp$ & $\mathrm{f}$ & $\mathbf{t}$ & $\mathrm{t}$ & $T$ & $\mathbf{t}$ & $\mathrm{t}$ \\
\hline$\top$ & $\top$ & $\top$ & $T$ & $\top$ & $T$ & $\top$ & $\mathrm{T}$ & $T$ & $\top$ & $T$ & $T$ \\
\hline$\perp$ & $\perp$ & $\perp$ & $\perp$ & $\top$ & $\perp$ & f & $\perp$ & $\mathbf{t}$ & $T$ & $\perp$ & $\perp$ \\
\hline f & $\mathbf{t}$ & f & f & $\top$ & f & f & f & $\mathbf{t}$ & $\top$ & $\perp$ & $\mathbf{f}$ \\
\hline
\end{tabular}

Figure 6: Truth-tables for the logic $\mathrm{dS}_{\text {fde }}$

Observation 1. $\mathrm{dS}_{\mathrm{fde}}$ is a Dual Parry logic.

Proof. Suppose, for reductio, that there is an inference $\Gamma \vDash_{\mathrm{dS}_{\mathrm{fde}}} \varphi$ such that it is not true that $\exists \Gamma^{\prime} \subseteq \Gamma, \Gamma^{\prime} \neq \emptyset, \operatorname{var}\left(\Gamma^{\prime}\right) \subseteq \operatorname{var}(\varphi)$. Notice, furthermore, that for all $\psi$ there is a valuation $v$ such that for all $p \in \operatorname{var}(\psi), v(p)=\perp$, whence $v(\psi)=\perp \notin \mathcal{D}_{\mathrm{dS}_{\mathrm{fde}}}$. Consider a valuation $v$ of this sort, where $v(\varphi)=\perp \notin \mathcal{D}_{\mathrm{dS}_{\mathrm{fde}}}$, to construct the valuation $v^{*}$

$$
v^{*}(p)= \begin{cases}\top & \text { if } p \in \operatorname{var}(\Gamma) \backslash \operatorname{var}(\varphi) \\ v(p) & \text { otherwise }\end{cases}
$$

By the above, we know for all $\gamma \in \Gamma, \operatorname{var}(\gamma) \nsubseteq \operatorname{var}(\varphi)$, which implies for all $\gamma \in \Gamma$, there is a $q \in \operatorname{var}(\gamma) \backslash \operatorname{var}(\varphi)$ such that $v^{*}(q)=\top$. Whence, for all $\gamma \in \Gamma, v^{*}(\gamma)=\top$, implying $v^{*}(\Gamma) \subseteq$ $\mathcal{D}_{\mathrm{dS}_{\text {fde }}}$. Simultaneously, by our assumptions, $v^{*}(\varphi) \notin \mathcal{D}_{\mathrm{dS}_{\text {fde }}}$. Then, $v^{*}$ witnesses $\Gamma \not \nvdash_{\mathrm{dS}_{\mathrm{fde}}} \varphi$, contradicting our initial assumption. Therefore, $\mathrm{dS}_{\mathrm{fde}}$ is a Dual Parry logic.

Observation 2. Letting $\Sigma\urcorner=\{\neg \psi \mid \psi \in \Sigma\}$, the following readily verifiable fact establishes the duality between $\mathrm{dS}_{\text {fde }}$ and Deutsch's logic $\mathrm{S}_{\text {fde }}$

$$
\left.\Gamma \vDash_{\mathrm{S}_{\text {fde }}} \Delta \Longleftrightarrow \Delta\right\urcorner \vDash_{\mathrm{dS}_{\text {fde }}} \Gamma^{\urcorner}
$$

Moreover, the logic $\mathrm{dS}_{\text {fde }}^{w}$, presented first in [13], is also a Dual Parry logic. This can be established - once again - by noting, as we do below, that $d S_{\text {fde }}^{w}$ is a fragment of $d S_{\text {fde }}$.

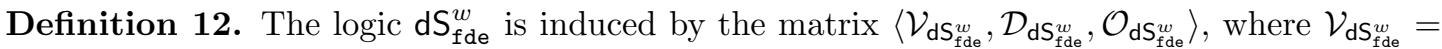
$\{\mathbf{t}, \top, \perp, \mathbf{f}\}, \mathcal{D}_{\mathrm{dS}_{\mathrm{fde}}^{w}}^{w}=\{\mathbf{t}, \top\}, \mathcal{O}_{\mathrm{dS}_{\mathrm{fde}}^{w}}^{w}=\left\{\dot{\overrightarrow{d S}}_{\mathrm{fde}}^{w}, \dot{\hat{d}}_{\mathrm{fde}}^{w}, \dot{\mathrm{d}}_{\mathrm{fde}}^{w}\right\}$ and these truth-functions are defined by

\begin{tabular}{|c|c|c|c|c|c|c|c|c|c|c|c|}
\hline & $\dot{\overrightarrow{\mathrm{d}}} \mathrm{S}_{\mathrm{fde}}^{w}$ & $\dot{\hat{d}}_{\mathrm{d} S_{\mathrm{fde}}^{w}}^{w}$ & $\mathrm{t}$ & $\top$ & $\perp$ & f & $\begin{array}{l}\dot{V} \\
d_{\text {fde }}^{w}\end{array}$ & $\mathrm{t}$ & $T$ & $\perp$ & f \\
\hline $\mathrm{t}$ & f & $\mathbf{t}$ & $\mathrm{t}$ & $T$ & $\perp$ & $\mathrm{f}$ & $\mathrm{t}$ & $t$ & $T$ & $\perp$ & $\mathrm{t}$ \\
\hline$\top$ & $\top$ & $\top$ & $T$ & $\top$ & $\top$ & $\top$ & $T$ & $\top$ & $T$ & $\top$ & $\top$ \\
\hline$\perp$ & $\perp$ & $\perp$ & $\perp$ & $\top$ & $\perp$ & $\perp$ & $\perp$ & $\perp$ & $T$ & $\perp$ & $\perp$ \\
\hline $\mathbf{f}$ & $\mathbf{t}$ & $f$ & $\mathbf{f}$ & $\top$ & $\perp$ & f & $f$ & $\mathbf{t}$ & $T$ & $\perp$ & f \\
\hline
\end{tabular}
the truth-tables in Figure 7.

Figure 7: Truth-tables for the logic $\mathrm{dS}_{\mathrm{fde}}^{w}$

That $d S_{\text {fde }}^{w}$ is a fragment of $d S_{\text {fde }}$ can be proved in the same way in which it was proved that $\mathrm{S}_{\mathrm{fde}}^{w}$ is a fragment of $\mathrm{S}_{\mathrm{fde}}$. For this purpose, the main argument carries over to this case, and so 
in fact we can check, by looking at the corresponding truth-tables, that $\underset{\mathrm{dS}_{\mathrm{fde}}^{w}}{\dot{w}}(x, y)$ is definable in $\mathrm{dS}_{\text {fde }}$ as

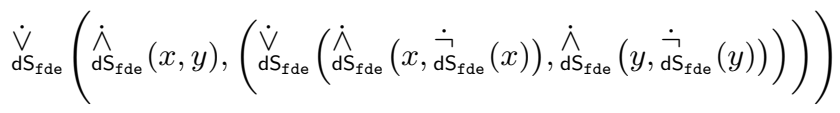

Next, we will focus on reviewing and showing some connections these containment systems have with bilattices, similar to those enjoyed by Belnap-Dunn logic $\mathrm{E}_{\mathrm{fde}}$ and bilattices.

\section{Logical Bilattices}

We now turn to logical systems based on bilattices.

Definition 13 (Arieli and Avron [2]). A bifilter on a bilattice $\mathcal{B}$ is a nonempty and proper subset $\mathcal{F} \subset B$ such that for all $a, b \in B: a \wedge b \in \mathcal{F}$ iff $a \in \mathcal{F}$ and $b \in \mathcal{F}$ iff $a \otimes b \in \mathcal{F}$. A bifilter $\mathcal{F}$ is prime if for all $a, b \in B: a \vee b \in \mathcal{F}$ iff either $a \in \mathcal{F}$ or $b \in \mathcal{F}$ iff $a \oplus b \in \mathcal{F}$.

Definition 14 (Arieli and Avron [2]). A logical bilattice is a pair $\langle\mathcal{B}, \mathcal{F}\rangle$ where $\mathcal{B}$ is a bilattice and $\mathcal{F}$ is a prime bifilter on $\mathcal{B}$.

Observation 3 (Arieli and Avron [2]). The set $\{\mathbf{t}, \top\}$ is the only prime bifilter on the bilattice $\mathcal{F O U R}$. Thus, the only logical bilattice definable on $\mathcal{F O U R}$ is $\langle\mathcal{F O U R},\{\mathbf{t}, \top\}\rangle$.

The key to defining different consequence relations on logical bilattices is the notion of a valuation based on a bilattice. Arieli and Avron, e.g. opted for the following.

Definition 15 (Arieli and Avron [1]). An Arieli-Avron valuation ( $A A$-valuation) on a bilattice $\mathcal{B}$ is a function $v: F O R(\mathcal{L}) \longrightarrow B$ interpreting the connectives in $\{\dot{\neg}, \dot{\wedge}, \dot{\vee}\}$ with the operations in $\{\neg, \wedge, \vee\}$, respectively.

Definition 16 (Arieli and Avron [1]). With regard to a logical bilattice $\langle\mathcal{B}, \mathcal{F}\rangle$, an inference from $\Gamma$ to $\varphi$ is $A A$-valid (symbolized $\Gamma \vDash_{\mathrm{AA}}^{\langle\mathcal{B}, \mathcal{F}\rangle} \varphi$ ) if for all $A A$-valuations $v$ such that $v(\Gamma) \subseteq \mathcal{F}$, then also $v(\varphi) \in \mathcal{F}$.

Thus, Arieli and Avron proved the following, interpreted by them as showing that $E_{f d e}$ is the logic of logical bilattices - a fortiori establishing that $\mathrm{E}_{\mathrm{fde}}$ has a similar relation to logical bilattices than Boolean algebras have with Classical Logic.

Fact 4 (Arieli and Avron [1]). For all logical bilattices $\langle\mathcal{B}, \mathcal{F}\rangle$ and all sets of formulae $\Gamma \cup\{\varphi\}$,

$$
\Gamma \vDash_{\mathrm{AA}}^{\langle\mathcal{B}, \mathcal{F}\rangle} \varphi \Longleftrightarrow \Gamma \vDash_{\mathrm{E}_{\text {fde }}} \varphi
$$

Generalizing Fitting's work, Ferguson focused in [7] on different definitions inspired by the "cut-down" interpretation, proving similar results about the relation of $\mathrm{S}_{\text {fde }}$ and bilattices.

Definition 17 (Ferguson [7]). A Kleene-Fitting valuation on a bilattice $\mathcal{B}$ is a function $v$ : $F O R(\mathcal{L}) \longrightarrow B$ interpreting the connectives in $\{\dot{\neg}, \dot{\wedge}, \dot{\vee}\}$ with the operations in $\{\neg, \Delta, \nabla\}$, respectively.

Definition 18 (Ferguson [7]). With regard to a logical bilattice $\langle\mathcal{B}, \mathcal{F}\rangle$, an inference from $\Gamma$ to $\varphi$ is $K F$-valid (symbolized $\Gamma \models_{\mathrm{KF}}^{\langle\mathcal{B}, \mathcal{F}\rangle} \varphi$ ) if for all Kleene-Fitting valuations $v$ such that $v(\Gamma) \subseteq \mathcal{F}$, then also $v(\varphi) \in \mathcal{F}$.

This allowed him to prove the next result. 
Fact 5 (Ferguson [7]). For all logical bilattices $\langle\mathcal{B}, \mathcal{F}\rangle$ and all sets of formulae $\Gamma \cup\{\varphi\}$,

$$
\Gamma \vDash_{\mathrm{KF}}^{\langle\mathcal{B}, \mathcal{F}\rangle} \varphi \Longleftrightarrow \Gamma \vDash_{\mathrm{S}_{\mathrm{fde}}} \varphi
$$

Ferguson took this fact to establish that $S_{\text {fde }}$ is the logic of cut-down operations on logical bilattices and, hence, that $S_{\text {fde }}$ has the same relation with bilattices equipped with cut-down operations than Classical Logic has with Boolean algebras.

\section{The Logic of Track-Down Operations}

In this section we prove a result similar to the previously discussed about $\mathrm{E}_{\text {fde }}$ and $\mathrm{S}_{\text {fde }}$, but we now focus on $\mathrm{dS}_{\text {fde }}$.

Definition 19. A track-down valuation on a bilattice $\mathcal{B}$ is a function $v: F O R(\mathcal{L}) \longrightarrow B$ interpreting the connectives in $\{\dot{\neg}, \dot{\wedge}, \dot{V}\}$ with the operations in $\{\neg, \mathbf{\Lambda}, \mathbf{\nabla}\}$, respectively.

Definition 20. With regard to a logical bilattice $\langle\mathcal{B}, \mathcal{F}\rangle$, an inference from $\Gamma$ to $\varphi$ is $T D$ valid (symbolized $\Gamma \vDash_{\mathrm{TD}}^{\langle\mathcal{B}, \mathcal{F}\rangle} \varphi$ ) if for all track-down valuations $v$ such that $v(\Gamma) \subseteq \mathcal{F}$, then also $v(\varphi) \in \mathcal{F}$.

Observation 6. For all sets of formulae $\Gamma \cup\{\varphi\}$,

$$
\Gamma \vDash_{\mathrm{TD}}^{\langle\mathcal{F O U R},\{\mathbf{t}, T\}\rangle} \varphi \Longleftrightarrow \Gamma \vDash_{\mathrm{dS}_{\mathrm{fde}}} \varphi
$$

Proof. This follows straightforwardly, by identifying, one the one hand, the sets $\mathcal{V}_{\mathrm{dS}_{\text {fde }}}$ and $F O U R=\{\mathbf{t}, \top, \perp, \mathbf{f}\}$ and, on the other hand, the truth-functions of $\mathrm{dS}_{\text {fde }}$ with the corresponding track-down operations on $\mathcal{F} \mathcal{O U} \mathcal{R}$.

Definition 21 (Arieli and Avron [2]). Let $\langle\mathcal{B}, \mathcal{F}\rangle$ be a logical bilattice, we define the following exclusive and exhaustive subsets of $B$ :

$$
\begin{aligned}
& \mathcal{T}_{\mathbf{t}}^{\langle\mathcal{B}, \mathcal{F}\rangle}=\{a \in B \mid a \in \mathcal{F}, \neg a \notin \mathcal{F}\} \\
& \mathcal{T}_{\mathbf{T}}^{\langle\mathcal{B}, \mathcal{F}\rangle}=\{a \in B \mid a \in \mathcal{F}, \neg a \in \mathcal{F}\} \\
& \mathcal{T}_{\perp}^{\langle\mathcal{B}, \mathcal{F}\rangle}=\{a \in B \mid a \notin \mathcal{F}, \neg a \notin \mathcal{F}\} \\
& \mathcal{T}_{\mathbf{f}}^{\langle\mathcal{B}, \mathcal{F}\rangle}=\{a \in B \mid a \notin \mathcal{F}, \neg a \in \mathcal{F}\}
\end{aligned}
$$

Definition 22 (Arieli and Avron [2]). Let $\left\langle\mathcal{B}_{0}, \mathcal{F}_{0}\right\rangle$ and $\left\langle\mathcal{B}_{1}, \mathcal{F}_{1}\right\rangle$ be logical bilattices and let $a_{0} \in B_{0}$ and $a_{1} \in B_{1}$ be elements of each. Then $a_{0} \in B_{0}$ and $a_{1} \in B_{1}$ are similar $\left(a_{0} \simeq a_{1}\right)$ if: (i) $a_{0} \in \mathcal{F}_{0}$ iff $a_{1} \in \mathcal{F}_{1}$, and (ii) $\neg a_{0} \in \mathcal{F}_{0}$ iff $\neg a_{1} \in \mathcal{F}_{1}$.

Definition 23. Two track-down valuations $v_{0}$ and $v_{1}$ for logical bilattices $\left\langle\mathcal{B}_{0}, \mathcal{F}_{0}\right\rangle$ and $\left\langle\mathcal{B}_{1}, \mathcal{F}_{1}\right\rangle$, respectively, are similar $\left(v_{0} \simeq v_{1}\right)$ if for all atomic $p \in F O R(\mathcal{L}): v_{0}(p) \simeq v_{1}(p)$.

Proposition 7. Let $v_{0}$ and $v_{1}$ be two track-down valuations for logical bilattices $\left\langle\mathcal{B}_{0}, \mathcal{F}_{0}\right\rangle$ and $\left\langle\mathcal{B}_{1}, \mathcal{F}_{1}\right\rangle$, respectively. If $v_{0} \simeq v_{1}$, then for all formulae $\varphi \in F O R(\mathcal{L}): v_{0}(\varphi) \simeq v_{1}(\varphi)$.

Proof. By induction on the complexity of $\varphi$.

Definition 24 (Ferguson [7]). Let $g_{\langle\mathcal{B}, \mathcal{F}\rangle}: B \longrightarrow F O U R$ be a function such that $g_{\langle\mathcal{B}, \mathcal{F}\rangle}(x)$ is the unique $y$ for which $x \in \mathcal{T}_{y}^{\langle\mathcal{B}, \mathcal{F}\rangle}$. 
What $g$ essentially does is to find the unique member of the partition provided in Definition 21, to which $x$ belongs, and then maps it to the corresponding element of FOUR.

Proposition 8. If $v$ is a track-down valuation on a logical bilattice $\langle\mathcal{B}, \mathcal{F}\rangle$, then $g_{\langle\mathcal{B}, \mathcal{F}\rangle} \circ v$ is a track-down valuation on $\langle\mathcal{F O U \mathcal { R } ,}\{\mathbf{t}, \top\}\rangle$ such that $v \simeq g_{\langle\mathcal{B}, \mathcal{F}\rangle} \circ v$.

Proof. Immediate from the definition of $g_{\langle\mathcal{B}, \mathcal{F}\rangle}$.

Lemma 9. For all logical bilattices $\langle\mathcal{B}, \mathcal{F}\rangle$ and all sets of formulae $\Gamma \cup\{\varphi\}$,

$$
\Gamma \vDash_{\mathrm{TD}}^{\langle\mathcal{B}, \mathcal{F}\rangle} \varphi \Longleftrightarrow \Gamma \vDash_{\mathrm{TD}}^{\langle\mathcal{F O U R},\{\mathbf{t}, \top\}\rangle} \varphi
$$

Proof. RTL: suppose $\Gamma \nvdash_{\mathrm{TD}}^{\langle\mathcal{B}, \mathcal{F}\rangle} \varphi$ and let $v$ be a track-down valuation on $\langle\mathcal{B}, \mathcal{F}\rangle$ witnessing this fact, i.e. $v(\Gamma) \subseteq \mathcal{F}$ and $v(\varphi) \notin \mathcal{F}$. By Proposition $8, g_{\langle\mathcal{B}, \mathcal{F}\rangle} \circ v$ is a track-down valuation on $\langle\mathcal{F O U R},\{\mathbf{t}, \top\}\rangle$ behaving in the following way: $g_{\langle\mathcal{B}, \mathcal{F}\rangle} \circ v(\Gamma) \subseteq\{\mathbf{t}, \top\}$ and $g_{\langle\mathcal{B}, \mathcal{F}\rangle} \circ v(\varphi) \notin\{\mathbf{t}, \top\}$. This implies $\Gamma \not \nvdash_{\mathrm{TD}}^{\langle\mathcal{F O U R},\{\mathbf{t}, T\}\rangle} \varphi$.

LTR: suppose $\Gamma \not \nvdash_{\mathrm{TD}}^{\langle\mathcal{F O U R},\{\mathbf{t}, T\}\rangle} \varphi$ and let $v$ be a track-down valuation on $\langle\mathcal{F O U \mathcal { R }},\{\mathbf{t}, \top\}\rangle$ witnessing this fact, i.e. $v(\Gamma) \subseteq\{\mathbf{t}, \top\}$ and $v(\varphi) \notin\{\mathbf{t}, \top\}$. Given $F O U R \subseteq B$, for all bilattices $\langle\mathcal{B}, \mathcal{F}\rangle$, we know that $v$ is a valuation on $\langle\mathcal{B}, \mathcal{F}\rangle$ too. Moreover, $v$ on $\langle\mathcal{B}, \mathcal{F}\rangle$ is similar to $v$ on $\langle\mathcal{F O U R},\{\mathbf{t}, \top\}\rangle$, since $\top, \mathbf{t} \in \mathcal{F}$ and $\perp, \mathbf{f} \notin \mathcal{F}$. Thus, $v$ witnesses $\Gamma \not \nvdash_{\mathrm{TD}}^{\langle\mathcal{B}, \mathcal{F}\rangle} \varphi$.

Theorem 10. For all logical bilattices $\langle\mathcal{B}, \mathcal{F}\rangle$ and all sets of formulae $\Gamma \cup\{\varphi\}$,

$$
\Gamma \vDash_{\mathrm{TD}}^{\langle\mathcal{B}, \mathcal{F}\rangle} \varphi \Longleftrightarrow \Gamma \vDash_{\mathrm{dS}_{\mathrm{fde}}} \varphi
$$

Proof. From Observation 6 and Lemma 9.

This establishes the first contribution of this paper, namely that $\mathrm{dS}_{\text {fde }}$ is the logic of trackdown operations on bilattices. This allows us to conclude - following Avron, Arieli and Fergusonthat the relation between $\mathrm{dS}_{\text {fde }}$ and bilattices endowed with track-down operations is the same that Boolean algebras have with Classical Logic.

Furthermore, this also facilitates the second contribution of this paper, consisting in an epistemic interpretation for Paraconsistent Weak Kleene. In fact, let us consider a situation in which we apply the track-down policy, but all experts consulted have determinate opinions on absolutely all propositions $\varphi$. This will amount, formally, to restricting the valuations of $\mathrm{dS}_{\text {fde }}$ to the values in $\{\mathbf{t}, \top, \mathbf{f}\}$. The three-valued logic induced by this restriction is no other than PWK, whence its operations can be interpreted in terms of the cut-down operations $\mathbf{\Lambda}, \mathbf{\nabla}$.

\section{Combined Cut-downs and Track-downs}

Finally, peculiar combinations of the cut-down and track-down approaches can, and perhaps should be conceived, for matters of exhaustivity. The combinations we are going to study next rely on applying the cut- and track-down approaches sequentially. By this we mean, defining cut-down operations which take the output of the track-down operations as an input, and viceversa.

To be more specific, concerning the cut-down approach, we could alternatively cut down experts who have expressed an opinion towards both $\varphi$ and $\psi$, taking into consideration the expert's opinion on $\varphi$ and $\psi$ only after the track-down process. That is, after tracking down whether their expressed an inconsistent opinion towards either $\varphi$ or $\psi$. This renders what we call the weak cut-down operations. 
Definition 25. For elements $a, b \in B$, the weak cut-down operations $\Delta^{w}$ and $\nabla^{w}$ are defined as:

$$
a \Delta^{w} b=(a \mathbf{\Delta} b) \otimes \llbracket a \rrbracket \otimes \llbracket b \rrbracket \quad a \nabla^{w} b=(a \mathbf{\nabla} b) \otimes \llbracket a \rrbracket \otimes \llbracket b \rrbracket
$$

Analogously, concerning the track-down approach, we could track down experts who have expressed an inconsistent opinion towards either $\varphi$ and $\psi$, taking into consideration the expert's opinion on $\varphi$ and $\psi$ only after the cut-down process. That is, after cutting down those experts which have not expressed an opinion towards either $\varphi$ or $\psi$. This renders what we call the weak track-down operations.

Definition 26. For elements $a, b \in B$, the weak track-down operations $\mathbf{\Delta}^{w}$ and $\boldsymbol{\nabla}^{w}$ are defined as:

$$
a \mathbf{\Delta}^{w} b=(a \triangle b) \oplus \rrbracket a \llbracket \oplus \rrbracket b \llbracket \quad a \nabla^{w} b=(a \nabla b) \oplus \rrbracket a \llbracket \oplus \rrbracket b \llbracket
$$

We show, next, some results concerning the relation between bilattices, $S_{\text {fde }}^{w}$ and $d S_{\text {fde }}^{w}$, which are equally conclusive than those previously discussed regarding $E_{f d e}, S_{\text {fde }}$ and $d S_{\text {fde }}$.

Definition 27. A weak cut-down valuation on a bilattice $\mathcal{B}$ is a function $v: F O R(\mathcal{L}) \longrightarrow B$ interpreting the connectives in $\{\dot{\neg}, \dot{\wedge}, \dot{\vee}\}$ with the operations in $\left\{\neg, \Delta^{w}, \nabla^{w}\right\}$, respectively.

Definition 28. With regard to a logical bilattice $\langle\mathcal{B}, \mathcal{F}\rangle$, an inference from $\Gamma$ to $\varphi$ is $C D^{w}$-valid (symbolized, $\Gamma \vDash_{\mathrm{CD}^{w}}^{\langle\mathcal{B}, \mathcal{F}\rangle} \varphi$ ) if for all weak cut-down valuations $v$ such that $v(\Gamma) \subseteq \mathcal{F}$, then also $v(\varphi) \in \mathcal{F}$.

Definition 29. A weak track-down valuation on a bilattice $\mathcal{B}$ is a function $v: F O R(\mathcal{L}) \longrightarrow B$ interpreting the connectives in $\{\dot{\neg}, \dot{\wedge}, \dot{V}\}$ with the operations in $\left\{\neg, \mathbf{\Lambda}^{w}, \boldsymbol{\nabla}^{w}\right\}$, respectively.

Definition 30. With regard to a logical bilattice $\langle\mathcal{B}, \mathcal{F}\rangle$, an inference from $\Gamma$ to $\varphi$ is $T D^{w}$-valid (symbolized, $\Gamma \vDash_{\mathrm{TD}^{w}}^{\langle\mathcal{B}, \mathcal{F}\rangle} \varphi$ ) if for all weak track-down valuations $v$ such that $v(\Gamma) \subseteq \mathcal{F}$, then also $v(\varphi) \in \mathcal{F}$.

We can adapt the definitions and proofs of Section 5 to show that $S_{\text {fde }}^{w}$ and $d S_{\text {fde }}^{w}$ are, respectively, the logic of weak cut-down and weak track-down operations on bilattices.

Observation 11. For all sets of formulae $\Gamma \cup\{\varphi\}$,

$$
\Gamma \vDash_{\mathrm{CD}^{w}}^{\langle\mathcal{F O U R},\{\mathbf{t}, T\}\rangle} \varphi \Longleftrightarrow \Gamma \vDash_{\mathrm{S}_{\mathrm{fde}}^{w}} \varphi
$$

Proof. We establish this by identifying the sets $\mathcal{V}_{\mathrm{S}_{\text {fde }}^{w}}$ and $F O U R=\{\mathbf{t}, \top, \perp$, f $\}$, and the truthfunctions of $S_{\text {fde }}^{w}$ with the corresponding weak cut-down operations on $\mathcal{F O U R}$.

Observation 12. For all sets of formulae $\Gamma \cup\{\varphi\}$,

$$
\Gamma \vDash_{\mathrm{TD} w}^{\langle\mathcal{F} \mathcal{O} \mathcal{R},\{\mathbf{t}, T\}\rangle} \varphi \Longleftrightarrow \Gamma \vDash_{\mathrm{dS}_{\mathrm{fde}}^{w}}^{w} \varphi
$$

Proof. For this, we identify the sets $\mathcal{V}_{\mathrm{dS}}^{\text {fde }}$ and $F O U R=\{\mathbf{t}, \top, \perp, \mathbf{f}\}$, and the truth-functions of $\mathrm{dS}_{\text {fde }}^{w}$ with the corresponding weak track-down operations on $\mathcal{F} \mathcal{O U} \mathcal{R}$.

Definition 31. Two weak cut-down (alternatively, weak track-down) valuations $v_{0}$ and $v_{1}$ for logical bilattices $\left\langle\mathcal{B}_{0}, \mathcal{F}_{0}\right\rangle$ and $\left\langle\mathcal{B}_{1}, \mathcal{F}_{1}\right\rangle$, respectively, are similar $\left(v_{0} \simeq v_{1}\right)$ if for all atomic $p \in \operatorname{FOR}(\mathcal{L}): v_{0}(p) \simeq v_{1}(p)$. 
The following are proved exactly like in Section 5 .

Proposition 13. Let $v_{0}$ and $v_{1}$ be two weak cut-down (alternatively, weak track-down) valuations for logical bilattices $\left\langle\mathcal{B}_{0}, \mathcal{F}_{0}\right\rangle$ and $\left\langle\mathcal{B}_{1}, \mathcal{F}_{1}\right\rangle$, respectively. If $v_{0} \simeq v_{1}$, then for all formulae $\varphi \in F O R(\mathcal{L}): v_{0}(\varphi) \simeq v_{1}(\varphi)$.

Proposition 14. If $v$ is a weak cut-down (alternatively, weak track-down) valuation on a logical bilattice $\langle\mathcal{B}, \mathcal{F}\rangle$, then $g_{\langle\mathcal{B}, \mathcal{F}\rangle} \circ v$ is a weak cut-down (respectively, weak track-down) on

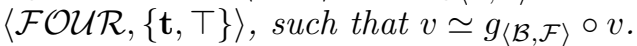

Lemma 15. For all logical bilattices $\langle\mathcal{B}, \mathcal{F}\rangle$ and all sets of formulae $\Gamma \cup\{\varphi\}$,

$$
\Gamma \vDash_{\mathrm{CD}^{w}}^{\langle\mathcal{B}, \mathcal{F}\rangle} \varphi \Longleftrightarrow \Gamma \vDash_{\mathrm{CD}^{w}}^{\langle\mathcal{F} \mathcal{U} \mathcal{R},\{\mathbf{t}, \top\}\rangle} \varphi
$$

Proof. RTL: suppose $\Gamma \not \nvdash_{\mathrm{CD}^{w}}^{\langle\mathcal{B}\rangle} \varphi$ and let $v$ be a weak cut-down valuation on $\langle\mathcal{B}, \mathcal{F}\rangle$ witnessing this fact, i.e. $v(\Gamma) \subseteq \mathcal{F}$ and $v(\varphi) \notin \mathcal{F}$. By Proposition 14, $g_{\langle\mathcal{B}, \mathcal{F}\rangle} \circ v$ is a weak cut-down valuation on $\langle\mathcal{F} \mathcal{O U} \mathcal{R},\{\mathbf{t}, \top\}\rangle$ behaving in the following way: $g_{\langle\mathcal{B}, \mathcal{F}\rangle} \circ v(\Gamma) \subseteq\{\mathbf{t}, \top\}$ and $g_{\langle\mathcal{B}, \mathcal{F}\rangle} \circ v(\varphi) \notin\{\mathbf{t}, \top\}$. This implies $\Gamma \not \nvdash_{\mathrm{CD}^{w}}^{\langle\mathcal{F O U R},\{\mathbf{t}, \top\}\rangle} \varphi$.

LTR: suppose $\Gamma \not \nvdash_{\mathrm{CD}^{w}}^{\langle\mathcal{F} \mathcal{U} \mathcal{R},\{\mathbf{t}, \top\}\rangle} \varphi$ and let $v$ be a weak cut-down valuation on $\langle\mathcal{F} \mathcal{O U} \mathcal{R},\{\mathbf{t}, \top\}\rangle$ witnessing this fact, i.e. $v(\Gamma) \subseteq\{\mathbf{t}, \top\}$ and $v(\varphi) \notin\{\mathbf{t}, \top\}$. Given $F O U R \subseteq B$, for all $\langle\mathcal{B}, \mathcal{F}\rangle$, we know $v$ is a valuation on $\langle\mathcal{B}, \mathcal{F}\rangle$ too. Moreover, $v$ on $\langle\mathcal{B}, \mathcal{F}\rangle$ is similar to $v$ on $\langle\mathcal{F} \mathcal{O U} \mathcal{R},\{\mathbf{t}, \top\}\rangle$, since $\top, \mathbf{t} \in \mathcal{F}$ and $\perp, \mathbf{f} \notin \mathcal{F}$. Thus, $v$ witnesses $\Gamma \not \nvdash_{\mathrm{CD}^{w}}^{\langle\mathcal{B}, \mathcal{F}\rangle} \varphi$.

Lemma 16. For all logical bilattices $\langle\mathcal{B}, \mathcal{F}\rangle$ and all sets of formulae $\Gamma \cup\{\varphi\}$,

$$
\Gamma \vDash{ }_{\mathrm{TD}^{w}}^{\langle\mathcal{B}, \mathcal{F}\rangle} \varphi \Longleftrightarrow \Gamma \vDash_{\mathrm{TD}^{w}}^{\langle\mathcal{F O U R},\{\mathbf{t}, \top\}\rangle} \varphi
$$

Proof. Similar to the proof of Lemma 15, replacing "weak cut-down valuation" for "weak trackdown valuation".

Theorem 17. For all logical bilattices $\langle\mathcal{B}, \mathcal{F}\rangle$ and all sets of formulae $\Gamma \cup\{\varphi\}$,

$$
\Gamma \vDash_{\mathrm{CD}^{w}}^{\langle\mathcal{B}, \mathcal{F}\rangle} \varphi \Longleftrightarrow \Gamma \vDash_{\mathrm{S}_{\mathrm{fde}}^{w}} \varphi
$$

Proof. From Observation 11 and Lemma 15.

Theorem 18. For all logical bilattices $\langle\mathcal{B}, \mathcal{F}\rangle$ and all sets of formulae $\Gamma \cup\{\varphi\}$,

$$
\Gamma \vDash_{\mathrm{TD}^{w}}^{\langle\mathcal{B}, \mathcal{F}\rangle} \varphi \Longleftrightarrow \Gamma \vDash_{\mathrm{dS}_{\mathrm{fde}}^{w}} \varphi
$$

Proof. From Observation 12 and Lemma 16.

\section{$7 \quad$ Further Research}

A proof-theoretic exploration of the logics of cut-down and track-down operations is pending. It appears straightforward to build Gentzen-style sequent calculi for $\mathrm{dS}_{\text {fde }}$ and $\mathrm{S}_{\text {fde }}$, using the techniques described in [4], by imposing variable inclusion restrictions on the rules - respectively - of the sequent calculi for $\mathrm{K}_{3}$ and its paraconsistent dual LP. However, we conjecture special care needs to be taken in trying to reflect, in potential calculi for $S_{\text {fde }}^{w}$ and $d S_{\text {fde }}^{w}$, the sequential nature of the combinations of cut- and track-downs behind these systems. 


\section{References}

[1] O. Arieli and A. Avron. Reasoning with logical bilattices. Journal of Logic, Language and Information, 5(1):25-63, 1996.

[2] O. Arieli and A. Avron. The logical role of the four-valued bilattice. In Proc. 13th Symposium on Logic in Computer Science, pages 118-126, Los Alamitos, CA, 1998. IEEE Computer Society.

[3] N. Belnap. A useful four-valued logic. In M. Dunn and G. Epstein, editors, Modern uses of Multiple-Valued Logic, pages 8-37. Reidel, Dordrecht, 1977.

[4] M. Coniglio and M. I. Corbalán. Sequent calculi for the classical fragment of Bochvar and Halldén's Nonsense Logics. In Proc. 7th Workshop on Logical and Semantic Frameworks with Applications (LSFA), pages 125-136. EPTCS 113, 2012.

[5] H. Deutsch. Relevant analytic entailment. The Relevance Logic Newsletter, 2(1):26-44, 1977.

[6] M. Dunn. Intuitive semantics for first-degree entailments and 'coupled trees'. Philosophical Studies, 29(3):149-168, 1976.

[7] T. M. Ferguson. Cut-Down Operations on Bilattices. In Proc. 45th International Symposium on Multiple-Valued Logic (ISMVL), pages 24-29, Los Alamitos, CA, 2015. IEEE Computer Society.

[8] T. M. Ferguson. Logics of nonsense and Parry systems. Journal of Philosophical Logic, $44(1): 65-80,2015$.

[9] M. Fitting. Bilattices are nice things. In T. Bolander, V. Hendricks, and S. Pedersen, editors, Self-Reference, pages 53-78. CSLI Publications, Stanford, 2006.

[10] M. Ginsberg. Multivalued logics: A uniform approach to reasoning in artificial intelligence. Computational Intelligence, 4(3):265-316, 1988.

[11] S. Gottwald. Many-valued logics. In D. Jacquette, editor, Philosophy of Logic, pages 675-722. North Holland, Amsterdam, 2007.

[12] W. T. Parry. Ein Axiomensystem für eine neue Art von Implikation (analytische Implikation). Ergebnisse eines mathematischen Kolloquiums, 4:5-6, 1933.

[13] D. Szmuc. Defining LFIs and LFUs in extensions of infectious logics. Journal of Applied Non-Classical Logics, 26(4):286-314, 2017. 